\title{
Automatic Measurement of Dimensions of 3D Foot Scan Data
}

\author{
Jinkyou SON*, Seung-Yeob BAEK, Kunwoo LEE \\ Human Centered CAD Laboratory, Seoul National University, Korea
}

\begin{abstract}
Recent trend of user-customization of the foot wears draws attention to the importance of digitized foot measurement due to its accuracy and efficiency. Conventional methods of measuring the foot dimensions, however, are not attractive in a sense that they usually take a lot of time for manually determining the landmark feature points, and that they are too much dependent on the subjective opinion of examiners and thus not reproducible. Also in the way to measure the foot dimensions from 3D scan data of a foot, landmarks still need to be manually determined by human investigator before or after scanning. Moreover, definitions of the foot dimensions and the way to measuring them are so much different from researchers to researchers so that it may confuse examiners to perform their tasks. In this paper, to solve these problems, we are to summarize previous works in literature and to propose the standards for measuring foot size dimensions. Further, by employing the computational geometry algorithms that automatically localizes the landmark points we overcome the limitations of the manual localization. As a result, an algorithm that takes raw 3D scan data of subject's foot and returns the locations of the landmark points and the virtually-measured foot dimensions. To examine our method, we tested 325 foot scan data that are randomly collected from our foot shape database. The algorithm aligned the data to have same position and orientation, and automatically found the landmark positions according to the geometric property. Then, the foot dimensions are measured by computing the lengths, circumferences, angles defined upon the landmarks. As a result we could find that those results are perfectly reproducible so that they are always the same for every trial. Further, we could also find that the algorithm localizes the landmark points consistently from model to model, and therefore it can be served as a standard foot measurement method. Moreover, since our method is fully automatic, it is needless to say that it took extremely short time and does not require any tiresome labor.
\end{abstract}

Keywords: Foot scanning, Automatic landmark localization, Simulated foot measurement

\section{Introduction}

User-tailored product design has been applied to many products in various industries recently. In particular, an effect of the foot health on the health of an entire body has become a rising issue, and this has caused a popularization of the personal fit footwear (shoes, insoles, foot orthotics, etc.).

In order to be comfortable, footwear needs to fit to the shape of a foot $[1,2,3]$. If not, a user would feel uncomfortable and his or her gait would be disturbed, which then would cause callosity, calluses, corns $[4,5]$, foot injuries $[6,7,8]$, etc. Conventionally, footwear has been mass produced based on just a foot length. However, foot length is not the only determining factor for a personal fit footwear, because the feet of a same length may have different shapes $[9,10]$. Therefore, many more measurement factors of a foot shape need to be considered to apply a foot shape into the footwear design with better accuracy $[11,12]$.

The most widely used method of designing user-tailored footwear has been based upon the foot measurements that are done manually. This is a cumbersome procedure because we need to find every landmarks of a foot to make measurements. This procedure is also not reproducible, because the landmarks can only be located subjectively and has low intra and inter-observer reliability [13].

With the recent improvement of reverse engineering, using 3D scanned data of foot captured from many foot related research has been another widely used method [14]. However, this still is a tedious procedure because it is not a fully digitized and automated method. When performing 3D scan, we need to attach markers on the foot landmarks beforehand [13,15], and manually indicate the landmarks on the 3D data after scanning $[14,16,17]$. This procedure is also not standardized in defining the foot landmarks and making measurements; hence, this method brings confusions among the researchers due to its subjectivity $[18,19]$.

This research analyzes and builds upon the previous research on the foot measurement, and proposes the standards in locating the foot landmarks in digital and automatic manner. Further, by employing the computational geometry algorithms that automatically localizes the landmark points, we were able to overcome the limitations of the existing methods of measuring foot dimensions mentioned above.

*flyingj@snu.ac.kr; +82-2-880-7447; hccl.snu.ac.kr 


\section{Previous Research}

\subsection{Alignment}

Before making any measurement, the alignment of $3 \mathrm{D}$ data needs to be done to make the measurements repeatable, because the position and the orientation of the 3D data points differ among each other. The alignment is really important because the value of measurements is dependent on the alignment method we used [20].

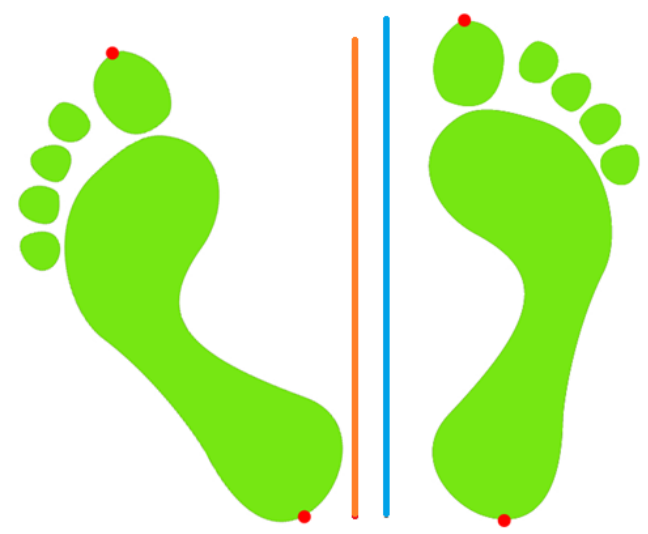

Fig. 1. An example of difference between dimensions by alignment.

There are four methods to align in general: Heel Centerline Method(HCL), Brannock Aligment Method(BRN), Pternion-Second Metatarsal Alignment Method(2MT), Medial Archline Method(MAL)[20]. The HCL method uses the base axis which passes through the pternion and the tip of the second toe[21]. The BRN method follows the process of Brannok device [22], a widely used method in the U.S. for measuring foot dimensions, which defines the base axis to be across the pternion and the point which is $38.1 \mathrm{~mm}$ apart from the first metatarsal head [14]. The base axis of the 2MT method is determined by joining the pternion and the second metatarsal[23]. The MAL method uses the medial arch line of the foot as the base axis.

Although the HCL method is the most commonly used alignment method, the alignment is very sensitive to a deformation of the second toe (for instance, second toe deformation affected by hallux varus) such that a small change in the second toe would result in a huge difference in the measurement and thus not robust; hence, this method is not preferable for the alignment [20]. Since the BRN method uses the constant value of $38.1 \mathrm{~mm}$ regardless of the foot sizes, it is dependent to the foot size differences, and so thus the alignment. As the 2MT method uses the second metatarsal as a reference, it is more independent to the foot shape deformation like hallux varus [20]; however, the second metatarsal is hard to observe by naked eye, and even harder to locate on 3D scan data. MAL method is easy to find the base axis and is scale independent because it uses medial arch line which is the most noticeable feature of a foot[24,25]. Therefore, we employ the MAL method for alignment due to its features metioned above.

\subsection{Landmarks}

Important foot-geometrical features that are used for various foot-measurement research include heel point, toe point, first metatarsal head $(1 \mathrm{MH})$, fifth metatarsal head $(5 \mathrm{MH})$, arch point, arch height point, dorsum, instep point, mid-foot point, heel lateral/medial points (See Figure 6, and Table 1).

Heel/toe point are points located at the very rear/front of the foot, whose locations can be trivially determined by finding the most posterior/frontal point in foot length direction [20]. In this reason, many foot alignment methods refer one or both of those points as geometric reference for the process.

$1 \mathrm{MH}$ and $5 \mathrm{MH}$ are one of the most prominent points that could be easily determined through naked eye. In this reason, many relevant researches use these points as landmark points $[13,16,17,26]$. Manually, these points are located by physical palpation of human investigator based on foot anatomy. Unfortunately, however, there is no obvious method for determining these points automatically, as 3D foot scan data lack information on internal anatomy. In this paper, we define the location of $1 \mathrm{MH}$ as a frontal tangent point on a medial bi-tangent line (medial arch line) (see Fig. 2). In addition, we define other remaining tangent point as a heel medial point. Similarly, we also define the location of $5 \mathrm{MH}$ as a frontal tangent point on a lateral bi-tangent line (lateral arch line). In the same way, the other remaining tangent point is defined as a heel lateral point. 
Such a way of determining those landmarks is much more robust than the previous methods that estimate $1 \mathrm{MH}$ and $5 \mathrm{MH}$ as the most protruded points on front foot, and consequently estimate heel medial and lateral points as the widest part on the heel parallel to the line connecting $1 \mathrm{MH}$ and $5 \mathrm{MH}$ $[16,17]$.

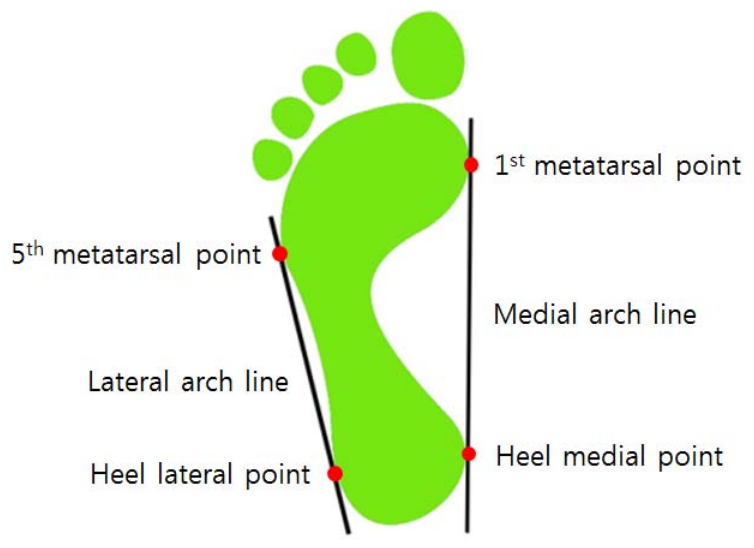

Fig. 2. The location of $1 \mathrm{MH}, 5 \mathrm{Mh}$, heel medial, heel lateral point and medial, lateral arch line.

Arch point is the farthest point on the medial arch area in footprint [24]. There are some variations of existing methods to find this point since the word "farthest" can be interpreted in many ways. In this paper, we define the arch point as the farthest point in foot width direction from the medial arch line, in order to improve robustness.

In general, arch height point is defined as the highest point on the instep surface at the location of $50 \%$ foot length (midfoot) [27]. This is fine for discriminating flatfoots from normal foots [27], but not suitable for designing the footwear, where the arch height is differently defined as the height of arch support. Therefore, to improve compatibility, we alternate the name 'arch height point' with 'midfoot height point'. Further, we newly define the arch height point as a highest point on a projected line of the medial arch line on the lower foot surface.

Dorsum point is a point defined on upper surface of the mid-foot whose definition varies controversially in many previous researches; in some cases it is defined as a point on talus where instep area and ankle intersect [13, 16, 19], while sometimes defined as a protruded point on navicular bone [27, 28]. In our opinion, it is better to define the dorsum point as a point on the talus bone as like the ones in the former cases, since navicular bone can hardly be distinguished on the 3D scanned surface. Based on this reason, we defined the dorsum point as a point having the biggest curvature on a curve passing through the ridge of instep area.

A list of the abovementioned landmark points and several additional landmarks can be found in Table 1. It is definitely clear the definitions of other landmark points that are not mentioned in this section, and hence we have omitted detailed discussion on them.
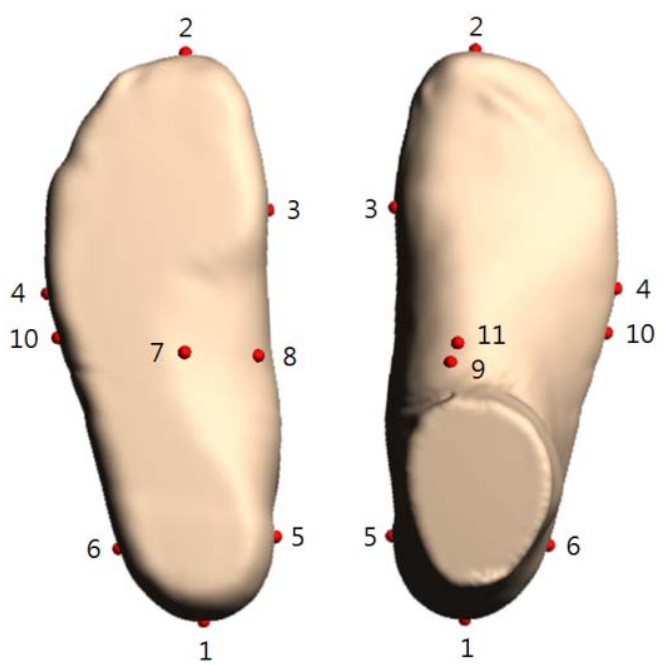

Fig. 3. A graphical illustration of the landmark points 


\section{Measuring Algorithm}

The data used in this paper are 3D foot scans of right feet of arbitrary subjects. The subjects are asked to wear socks and stand still on the scanner and to bear $50 \%$ of body weight on each foot during the scanning process. Since the same algorithm can be applied obviously to the left foot by mirroring the data, we do not discuss it separately in this paper.

\subsection{Alignment}

An algorithm for aligning 3D foot scan data in medial arch line direction is as follows:

Obtain footprint points by projecting the points whose distance from the ground is lesser than $3 \mathrm{~mm}$ on to ground plane.

Compute a 2D convex hull of above points. For computing the convex hull, several existing algorithms such as [29] can be employed.

From geometry of the footprint, the longest line segment on convex hull polygon is the medial arch line. We hence search for such a line by linearly exploring the line segments to find the medial arch line.

Rotate along the axis perpendicular to the ground to have $y$-direction parallel to the medial arch line.

Translate the data along the $y$-axis to have zero as the minimum y value. In addition, translate the data along the $x$-axis to have medial axis line collinear with the $y$-axis.

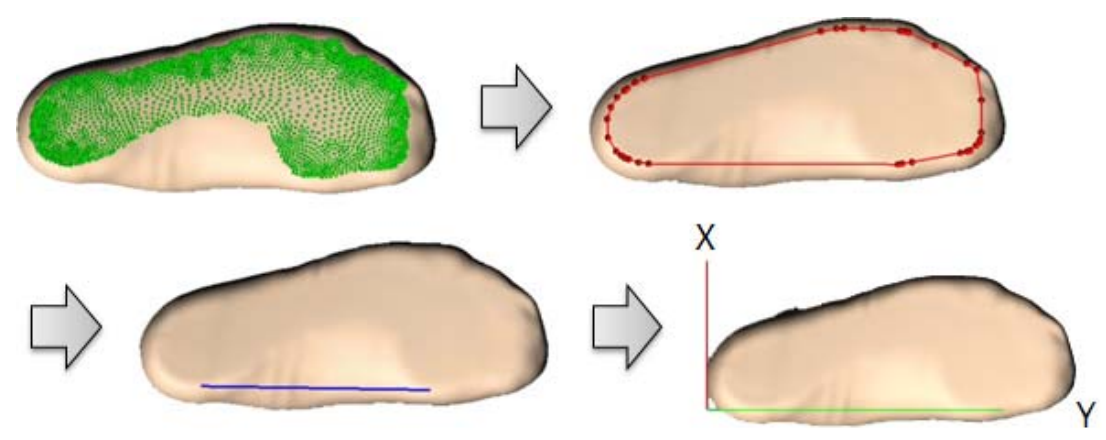

Fig. 4. Process of the alignment.

\subsection{Location of Landmarks}

After the alignment is done, the landmark points defined in section 2.2 is automatically located by the algorithm. This can be trivially done by following the definitions of the landmarks appeared in section 2.2. Following Table 1 lists the landmark points used in this paper and their rigorous mathematical definitions.

Table 1. Geometric landmarks used for the measurement of foot dimensions.

(1) Heel Point

(2) Toe Point

(3) $1 \mathrm{MH}$ Point

(4) $5 \mathrm{MH}$ Point

(5) Heel Medial Point

(6) Heel Lateral Point

(7) Arch Point

(8) Arch Height Point

(9) Dorsum Point

(10) Midfoot Lateral Point

(11) Midfoot Height Point
A point having the smallest $y$-coordinate value

A point having the greatest $y$-coordinate value

The end point of the medial arch

The end point of the lateral arch

The starting point of the medial arch line

The starting point of the lateral arch line

The most internal point of the arch boundary from the arch line

The highest point of a curve projected from the arch line to the foot surface.

An inflection point near the talus that is located around the intersection between the leg and the foot.

The point that has the greatest $x$-value at $50 \%$ of the foot length.

The point that has the greatest $z$-value at $50 \%$ of the foot length. 


\subsection{Measurement of dimensions}

There are many dimensions which can be measured from landmarks after alignment and location of landmarks. We measured 20 dimensions used generally in many researches as examples.

Table 2. Foot measurement items and their rules of calculation.

\begin{tabular}{ll}
\hline (1) Foot Length & $2 . y-1 . y$ \\
(2) 1 MH Length & $3 . y-1 . y$ \\
(3) 5 MH Length & $4 . y-1 . y$ \\
(4) Arch Length & $3 . y-5 . y$ \\
(5) Heel Length & $5 . y-1 . y$ \\
(6) Ball Width & $4 . x-3 . x$ \\
(7) Heel Width & $6 . x-8 . x$ \\
(8) Anatomical Ball Width & Dist(3,4) \\
(9) Anatomical Heel Width & Dist(5,6) \\
(10) Arch Width & $7 . x-3 . x$ \\
(11) Midfoot Width & $10 . x-3 . x$ \\
(12) Arch Height & $8 . z$ \\
(13) Dorsal Height & $9 . z$ \\
(14) Midfoot Height & $11 . z$ \\
(15) Ball Girth & Gir(3,4) \\
(16) Dorsal Girth & Gir(7, 11) \\
(17) Midfoot Girth & Gir(11, xz-plane) \\
(18) Ankle Girth & Gir(9, xy-plane) \\
(19) Footprint Angle & Ang(3-5, 3-7) \\
(20) Ball Angle & Ang(3-4, x-axis) \\
\hline
\end{tabular}

* the numbers in the right column denote the landmark indices in Table 1. Dist(a, b) is the distance between point $a$ and $b$. Gir( $a, b)$ is the girth of cross-sectional curve passes through point $a$ and $b$. Gir(a, b-plane)is the girth of cross-sectional curve passes point $a$ and is perpendicular to b-plane. Ang(a-b, c-d) is the angle between line a-b and line c-d. Ang(a-b, c-axis) is the angle between line a-b and c-axis.

\section{Test}

\subsection{Database}

We used a set of 3D scan data of 325 individuals that were obtained by a NEXCAN 3D foot scan device[30], which is a specially designed laser range scanner for the measurement of foot shapes. The scanner system is able to capture the entire shape of the foot. The scan data consists of almost 20,000 points and 40,000 facet saved in the *.ply format. Each individual was an adult residing in South Korea who was randomly chosen. All participants were requested to stand straight, with each of their feet bearing half of their body weight.

\subsection{Results}

Our algorithm took average time of $0.848 \mathrm{~s}$, with maximum of $1.465 \mathrm{~s}$, minimum of $0.197 \mathrm{~s}$, and the standard deviation was determined to be $0.226 \mathrm{~s}$. Table 3 . is results of measurement. 
Table 3. Results of measurement.

\begin{tabular}{|c|c|c|c|c|c|c|c|}
\hline Dimension & Avg & Min & Max & SD & $25 \%$ & $50 \%$ & $75 \%$ \\
\hline (1) & 253.4620 & 212.4967 & 285.5804 & 11.6923 & 246.9530 & 251.9911 & 262.0438 \\
\hline (2) & 146.3397 & 81.7716 & 189.8001 & 12.3726 & 139.3467 & 146.1584 & 153.0760 \\
\hline (3) & 186.0982 & 155.1624 & 232.9300 & 10.6722 & 179.1815 & 185.7634 & 192.1486 \\
\hline (4) & 142.4733 & 43.3587 & 181.7365 & 14.5430 & 135.0547 & 143.8973 & 151.1972 \\
\hline (5) & 99.9057 & 83.4691 & 113.4658 & 4.9313 & 96.5675 & 99.4461 & 102.9572 \\
\hline (6) & 66.8184 & 53.7386 & 104.4056 & 6.4327 & 63.1156 & 65.8418 & 69.2190 \\
\hline (7) & 95.7016 & 77.0519 & 109.8755 & 5.6136 & 91.8967 & 95.4983 & 99.3572 \\
\hline (8) & 256.6945 & 190.7001 & 306.9623 & 19.5189 & 248.7701 & 259.3194 & 268.7972 \\
\hline (9) & 259.5577 & 207.5600 & 347.3966 & 16.0760 & 250.1622 & 257.6107 & 267.0322 \\
\hline (10) & 256.3515 & 195.8686 & 293.5336 & 13.3337 & 248.3095 & 256.2515 & 263.5284 \\
\hline (11) & 269.5985 & 231.1281 & 352.9210 & 15.4708 & 259.0463 & 268.6795 & 279.7939 \\
\hline (12) & 171.5244 & 123.1610 & 211.8004 & 10.3046 & 165.5709 & 171.4847 & 177.7640 \\
\hline (13) & 68.7155 & 55.2213 & 130.7449 & 9.0481 & 64.3653 & 67.2741 & 70.5074 \\
\hline (14) & 107.1157 & 87.0106 & 170.6392 & 8.7478 & 101.9228 & 105.5606 & 110.9364 \\
\hline (15) & 81.5473 & 67.1747 & 100.6329 & 6.1498 & 77.1397 & 81.0526 & 85.5202 \\
\hline (16) & 256.1575 & 134.5151 & 340.4592 & 30.1747 & 243.5997 & 260.0578 & 273.3556 \\
\hline (17) & 28.9571 & 2.4766 & 83.1910 & 9.4421 & 24.7666 & 29.8781 & 35.2850 \\
\hline (18) & 15.6561 & 1.9339 & 52.0409 & 8.8437 & 10.2036 & 13.6549 & 17.9381 \\
\hline (19) & 19.6071 & 2.4891 & 43.5103 & 5.7412 & 17.3868 & 20.4105 & 23.2781 \\
\hline (20) & 24.2939 & 13.9124 & 63.3828 & 7.0825 & 19.6115 & 22.6403 & 27.2830 \\
\hline
\end{tabular}

* the numbers in the first column denote the dimensions indices in Table 2.

\section{Conclusion}

The goal of this research was to measure foot dimensions automatically from the 3D foot scan data, and to resolve the problems of investigator-dependency in other previous works. In this spirit, we have reorganized a set of landmark points used for measuring the dimensions and proposed clear and mathematically rigorous definitions of those landmarks that can be hopefully used as a standard in other future works.

To achieve so, we first had collected and surveyed many different kinds of foot alignment methods and then summarized them to derive a conclusion that the medial arch line alignment is the most robust and widely used algorithm. Consequently, based on this medial arch line based framework, we had surveyed various types of landmark points, which are defined in different manners from research to research even for the same landmark, and reorganized them to have the final 11 landmarks having rigorous mathematical definition based on the previous researches. Further, we developed an algorithm that implements the above alignment method and the landmark localization method for the 3D foot scan data. Using resultant landmark locations, we could then measure 20 foot dimensions defined on them.

As a result of our experiment, we could find out that our algorithm performs the same for every trials (reproducibility), takes extremely low time for the computation (efficiency), and automatically and directly measures the data from 3D foot scans without the need of human investigator (convenience). 
However, for some cases when the subject has severe foot disease that results geometric deformation of the foot shape, such as when the foot is extremely flat so that the medial arch line cannot be determined from the scan data or when the 1st toe is bent toward medio-lateral direction from severe hallux varus, our algorithm cannot be applied and still requires human investigator to manually and of course inaccurately and unreproducibly locate the landmark locations. But, it is so true that human foot shapes greatly differs from person to person, and it seems there could be no perfect algorithm that can be applied robustly regardless of the subjects' foot shapes.

Based on the research presented in this paper, our future work includes deeper geometry analysis on the foot shapes and the statistical shape analysis on the database. From those consequent researches, we expect to have detailed foot anthropometry data that can benefit various relevant industries.

\section{Acknowledgment}

This research was supported by a grant funded by the Small and Medium Business Administration of Korea.

This research was supported by Basic Science Research Program through the National Research Foundation of Korea (NRF) funded by the Ministry of Education, Science and Technology (2011-0001147).

This research project was supported by the Sports Promotion Fund of Seoul Olympic Sports Promotion Foundation from Ministry of Culture, Sports and Tourism.

\section{References}

1. Janisse, D. J., (1992): "The art and science of fitting shoes", Foot Ankle, Vol. 13, No. 5, pp. 257-262.

2. Goonetilleke, R. S., Luximon, A., Tsui, K. L., (2000): "The quality of footwear fit: what we know, don't know and should know", Proceedings of Human Factors and Ergonomics Society Conference 2000, San Diego, CA, Vol. 2, pp. 515-518.

3. Witana, C. P., Goonetilleke R. S., Feng, J., (2004): "Dimensional differences for evaluating the quality of footwear fit”, Ergonomics, Vol. 47, No. 12, pp. 1301-1317.

4. Singh, D., Bentley, G., Trevino, S. G., (1996): "Callosities, corns and calluses", British Medical Journal, Vol. 312, No. 1, pp. 1403-1406.

5. Watkins, P., J., (2003): "The diabetic foot", British Medical Journal, Vol. 326, No. 3, pp. 977-979

6. Hamill, J., Bates, B. T., Knutzen, K. M., Kirkpatrick, G. M., (1989): "Relationship between selected static and dynamic lower extremity measurers", Clinical Biomechanics, Vol. 4, pp. 217-225.

7. Messier, S. P., Davies, S. E., Curl, W. W., Lowery, R. B., Pack, R. J., (1991): "Etiology factors associated with patellofemoral pain in runners", Medicine and Science in Sports and Exercise, Vol. 23, pp. 1008-1015.

8. Frey, C., (2000): "Foot health and shoewear for women", Clinical Orthopaedics, Vol. 372, pp. 32-44.

9. Goonetilleke, R. S., Ho, E.C.F., So, R.H.Y., (1997): "Foot sizing beyond the 2-D Brannock method", Annual Journal of IIE, pp. 28-31.

10. Xiong, S., Goonetilleke, R. S., Witana C. P., Lee, A. E. Y., (2008): "Modelling foot height and foot shape-related dimensions", Ergonomics, Vol. 51, No. 8, pp. 1272-1289.

11. Rossi, W. Al., (1988): "The futile search for the perfect shoe fit", Journal of Testing and Evaluation, Vol. 16, No. 4, pp. 393-403.

12. Cheng, F. T., Perng, D. B., (1999): "A systematic approach for developing a foot size information system for shoe last design”, International Journal of Industrial Ergonomics, Vol. 25, pp.171-185.

13. Liu, W., Miller, J., Stefanyshyn, D., Nigg, B. M., (1999): "Accuracy and reliability of a technique for quantifying foot shape, dimensions and structural characteristics”, Ergonomics, Vol. 42, No. 2, pp. 346-358.

14. Mauch, M., Grau, S., Krauss, I., Maiwald, C., Horstmann, T., (2009): “A new approach to children's footwear based on foot type classification", Ergonomics, Vol. 52, No. 8, pp. 999-1008.

15. Witana, C. P., Xiong, S., Zhao, J., Goonetilleke, R. S, (2006): "Foot measurements from three-dimensional scans: A comparison and evaluation of different methods", International Journal of Industrial Ergonomics, Vol. 36, pp. 789-807.

16. Mauch M., Grau, S., Krauss, I., Maiwald, C., Horstmann, T., (2008): "Foot morphology of normal, underweight and overwight children", International Journal of Obesity, Vol. 32, pp. 1068-1075. 
17. Krauss, I., Langbein, C., Horstmann, T., and Grau, S., (2011): "Sex-related differences in foot shape of adult Caucasians - a follow-up study focusing on long and short feet", Ergonomics, Vol. 54, No. 3, pp. 294-300.

18. Hawes, M. R., Sovak, D., Miyashita M., Kang S. J., Yoshihuku, Y., Yanaka, S., (1994): "Ethnic differences in forefoot shape and the determination of shoe comfort", Ergonomics, Vol. 37, No. 1, pp. 187-196.

19. Hawes M. R., Sovak, D., (1994): "Quantitative morphology of the human foot in a North American population”, Ergonomics, Vol. 37, No. 7, pp. 1213-1226.

20. Goonetilleke, R. S., Witana, C. P., Zhao, J., Xiong, S., (2009): "The plus and minuses of obtaining measurements from digital scans", Digital Human Modeling, HCII 2009, LNCS 5620. pp. 681-690.

21. Kouchi, M., (2003): "Inter-generation differences in foot morphology: Aging or secular change?", Journal of Human Ergology, Vol. 32, pp. 23-48.

22. Foot measuring device "The Brannock Device", The Brannock Device Company, Liverpool, NY: www.brannock.com.

23. Yavatkar, A. S., (1993): "Computer aided system approach to determine the shoe-last size and shape based on statistical approximated model of a human foot", Unpublished master's thesis, Tufts University, Medford MA.

24. Cavanagh, P. R., Rodger, M. M., (1987): "The arch index: a useful measure from footprint", Journal of Biomechanics, Vol. 20, No. 5, pp. 547-551.

25. Shiang, T. Y., Lee, S. H., Lee, S. J., Chu, W. C., (1998): "Evaluating different footprint parameters as a predictor of arch height", Engineering in Medicine and Biology Society, Vol. 17, No. 6, pp. 62-66.

26. Rodrigo, A. S., Goonetilleke, R. S., (2009): "A turning function based approach for foot outline classification", Industrial Engineering and Engineering Management, pp. 861-864.

27. Williams, D. S., MaClay, I., S., (2000): "Measurements used to characterize the foot and the medial longitudinal arch: reliability and validity", Journal of the American Physical Therapy Association, Vol. 80, pp. 864-871.

28. Teyhen, D. S., et al., (2009): "Dynamic plantar pressure parameters associated with static arch height index during gait", Clinical Biomechanics, Vol. 24, pp. 391-396.

29. Jarvis, R. A., (1972): "On the identification of the convex hull of a finite set of points in the plane", Information Processing Letters, Vol. 2, pp. 18-21.

30. Foot scanning system "NEXCAN", K\&I Technology, Korea: www.knitech.co.kr. 